\title{
Dual delivery of active antibactericidal agents and bone morphogenetic protein at sustainable high concentrations using biodegradable sheath-core- structured drug-eluting nanofibers
}

\author{
This article was published in the following Dove Press journal: \\ International Journal of Nanomedicine \\ 17 August 2016 \\ Number of times this article has been viewed
}

\author{
Yung-Hen Hsu',2 \\ Chang-Tun Lin ${ }^{2}$ \\ Yi-Hsun $\mathrm{Yu}^{1,2}$ \\ Ying-Chao Chou ${ }^{1,2}$ \\ Shih-Jung Liu',2 \\ Err-Cheng Chan ${ }^{3}$ \\ 'Department of Orthopedic \\ Surgery, Chang Gung Memorial \\ Hospital, ${ }^{2}$ Department of Mechanical \\ Engineering, ${ }^{3}$ Department of Medical \\ Biotechnology and Laboratory \\ Science, School of Medical Technology, \\ Chang Gung University, Tao-Yuan, \\ Taiwan
}

Correspondence: Shih-Jung Liu Biomaterials Lab, Department of Mechanical Engineering, Chang Gung University, 259, Wen-Hwa Ist Road, Kwei-Shan, Tao-Yuan 333, Taiwan

Tel +88632118166

Fax +886 32118558

Email shihjung@mail.cgu.edu.tw

\begin{abstract}
In this study, we developed biodegradable sheath-core-structured drug-eluting nanofibers for sustainable delivery of antibiotics (vancomycin and ceftazidime) and recombinant human bone morphogenetic protein (rhBMP-2) via electrospinning. To prepare the biodegradable sheath-core nanofibers, we first prepared solutions of poly(D,L)-lactide-co-glycolide, vancomycin, and ceftazidime in 1,1,1,3,3,3-hexafluoro-2-propanol and rhBMP-2 in phosphate-buffered solution. The poly(D,L)-lactide-co-glycolide/antibiotics and rhBMP-2 solutions were then fed into two different capillary tubes controlled by two independent pumps for coaxial electrospinning. The electrospun nanofiber morphology was observed under a scanning electron microscope. We further characterized the in vitro antibiotic release from the nanofibers via high-performance liquid chromatography and that of rhBMP-2 via enzyme-linked immunosorbent assay and alkaline phosphatase activity. We showed that the biodegradable coaxially electrospun nanofibers could release high vancomycin/ceftazidime concentrations (well above the minimum inhibition concentration $[\mathrm{MIC}]_{90}$ ) and rhBMP-2 for $>4$ weeks. These experimental results demonstrate that novel biodegradable nanofibers can be constructed with various pharmaceuticals and proteins for long-term drug deliveries.
\end{abstract}

Keywords: biodegradable sheath-core nanofibers, coaxial electrospinning, vancomycin, ceftazidime, rhBMP-2, release characteristics

\section{Introduction}

Osteomyelitis in long bone fractures is often associated with severe soft tissue damage, bone defects, and ultimately nonunion. The mainstay of treatment for osteomyelitis comprises removal of the abscess and necrotic tissues, local and/or systemic administration of effective antibiotics against the infectious microorganism, and reconstruction of the injured bone or nonunion by bone grafting, bone transport, bone shortening, and/or implantation of artificial materials. ${ }^{1,2}$ Despite progress in surgical procedures and antimicrobial agents, osteomyelitis in long bone fractures presents a variety of challenges, including difficulty in controlling the infection, delay in bone reconstruction, and slow formation of new bone or callus during the repair process. Polymethylmethacrylate (PMMA) cement beads incorporating a high dose of antibiotics have been widely employed for osteomyelitis treatment and have proven useful. Such systems with sustained local antibiotic release are superior to parenteral administration because they deliver bactericidal concentrations of antibiotics directly to the target site while avoiding systemic toxicity. ${ }^{3}$ Nevertheless, the potential risk of irritating 
host tissues and the nondegradable nature of PMMA have limited its applications in osteomyelitis treatment and bone regeneration. A biodegradable and biocompatible delivery system that sustainably releases bone growth factors and antibiotics at levels well above bactericidal concentrations could provide an effective method for eradicating infection while promoting new local bone formation.

Bone morphogenetic proteins (BMPs), which are subgroups of growth factors, smartly regulate the bone healing process and tissue regeneration. ${ }^{4,5}$ BMPs also play as an important role in bone formation, and there is a need for new treatment strategies involving local delivery of growth factors to a bone defect site using carrier materials.

Furthermore, to treat osteomyelitis, surgeons must first identify the pathogens present and prescribe treatment with specific antimicrobial agents. ${ }^{6,7}$ Among current pharmaceuticals, vancomycin is widely employed to treat infections complicated by methicillin-resistant Staphylococcus aureus. Vancomycin-impregnated bone cements are commonly used in the prophylactic and therapeutic treatment for gram-positive infections. Ceftazidime (third-generation cephalosporin), on the other hand, exhibits strong activity against susceptible gram-negative bacteria and has been widely used to treat meningitis and infections of the lower respiratory tract, skin, urinary tract, bloodstream, joints, and abdomen.

In our previous study, we successfully developed antibiotic-eluting nanofibers for sustained release of vancomycin and ceftazidime to tissues surrounding bone screws and plates and demonstrated promising results in rabbits. ${ }^{8}$ Herein, we report the development of biodegradable sheath-core-structured drug-eluting nanofibers that co-deliver sustainable antibiotics (vancomycin/ceftazidime) and recombinant human BMP (rhBMP-2) using poly(D,L)lactide-co-glycolide (PLGA) as the carrier material. Among various polymeric materials available for a local release system, PLGA is a promising biodegradable material that allows sustained delivery of high antibiotic concentrations at the target site. ${ }^{9-13}$ The copolymer has also been approved for clinical applications owing to its nontoxicity, negligible inflammatory reaction, and biocompatible hydrolysis products (lactic and glycolic acids) after biodegradation. ${ }^{10,14,15}$

To prepare biodegradable sheath-core nanofibers, predetermined weight percentages of PLGA, vancomycin, and ceftazidime were dissolved in hexafluoroisopropyl alcohol (HFIP), and rhBMP-2 was dissolved in phosphate-buffered solution. The PLGA/antibiotics and rhBMP-2 solutions were then fed into two different capillary tubes with small-diameter needles for coaxial electrospinning. After electrospinning, the morphology of the spun nanofibers was examined by scanning electron microscope (SEM), and protein incorporation was verified using laser-scanning confocal microscopy. The antibiotics/rhBMP-2 release characteristics in vitro from the nanofibers were investigated using high-performance liquid chromatography (HPLC), a bacterial inhibition test, enzyme-linked immunosorbent assay (ELISA), and alkaline phosphatase (ALP) analysis.

\section{Materials and methods Fabrication of sheath-core-structured nanofibers}

Commercially available PLGA (Resomer RG 503), with a 50:50 lactide:glycolide ratio and a molecular weight (Mn) of 33,000 Da, was obtained from Sigma-Aldrich (St Louis, MO, USA). The antibiotics, vancomycin hydrochloride, and ceftazidime hydrate; the solvent for the polymers, HFIP; and powdered rhBMP-2 $(10 \mu \mathrm{g})$ were also purchased from Sigma-Aldrich.

We prepared two types of nanofibers: sheath-core antibiotics/BMP-2 nanofibers and blended antibiotics/BMP-2 nanofibers. To fabricate the sheath-core nanofibers, we designed and built a special coaxial device that delivers two polymer solutions simultaneously (Figure 1). Predetermined proportions of PLGA and antibiotics (250 mg PLGA, $20 \mathrm{mg}$ vancomycin, and $20 \mathrm{mg}$ ceftazidime) were dissolved in $1 \mathrm{~mL}$ HFIP and mixed by magnetic stirrer for 12 hours; in parallel, $25 \mu \mathrm{g}$ (or $100 \mu \mathrm{g}$ ) rhBMP-2 and $1 \mathrm{mg}$ BSA were dissolved in $1 \mathrm{~mL}$ phosphate-buffered solution. The PLGA and rhBMP-2 solutions were then loaded into two separate syringes with $0.42 \mathrm{~mm}$ diameter needles for coaxial electrospinning at room temperature. During spinning, the solutions were delivered by two independently controlled pumps with various volumetric flow rates: $0.9 \mathrm{~mL} / \mathrm{h}$ for the sheath PLGA/antibiotics solution and $0.3 \mathrm{~mL} / \mathrm{h}$ for the core rhBMP-2 solution. The solution in the syringe was set at a positive potential of $15 \mathrm{kV}$, and the travel distance for the ejected solution from the needle tip to the ground electrode for the fibers was $15 \mathrm{~cm}$. The thickness of the nanofibrous membrane thus obtained was $\sim 0.12 \mathrm{~mm}$.

We also prepared nanofibrous membranes of blended antibiotics/rhBMP-2/PLGA as a control. PLGA, vancomycin, ceftazidime, and rhBMP-2 (250 mg, $20 \mathrm{mg}, 20 \mathrm{mg}$, and $25 \mu \mathrm{g}$, respectively) were dissolved in $1 \mathrm{~mL}$ HFIP and loaded into a single syringe for electrospinning.

\section{Scanning electron microscope observation}

The morphology of electrospun nanofibers was observed on a field emission scanning electron microscope (JEOL Model JSM-7500F, Tokyo, Japan) after gold coating. The average 

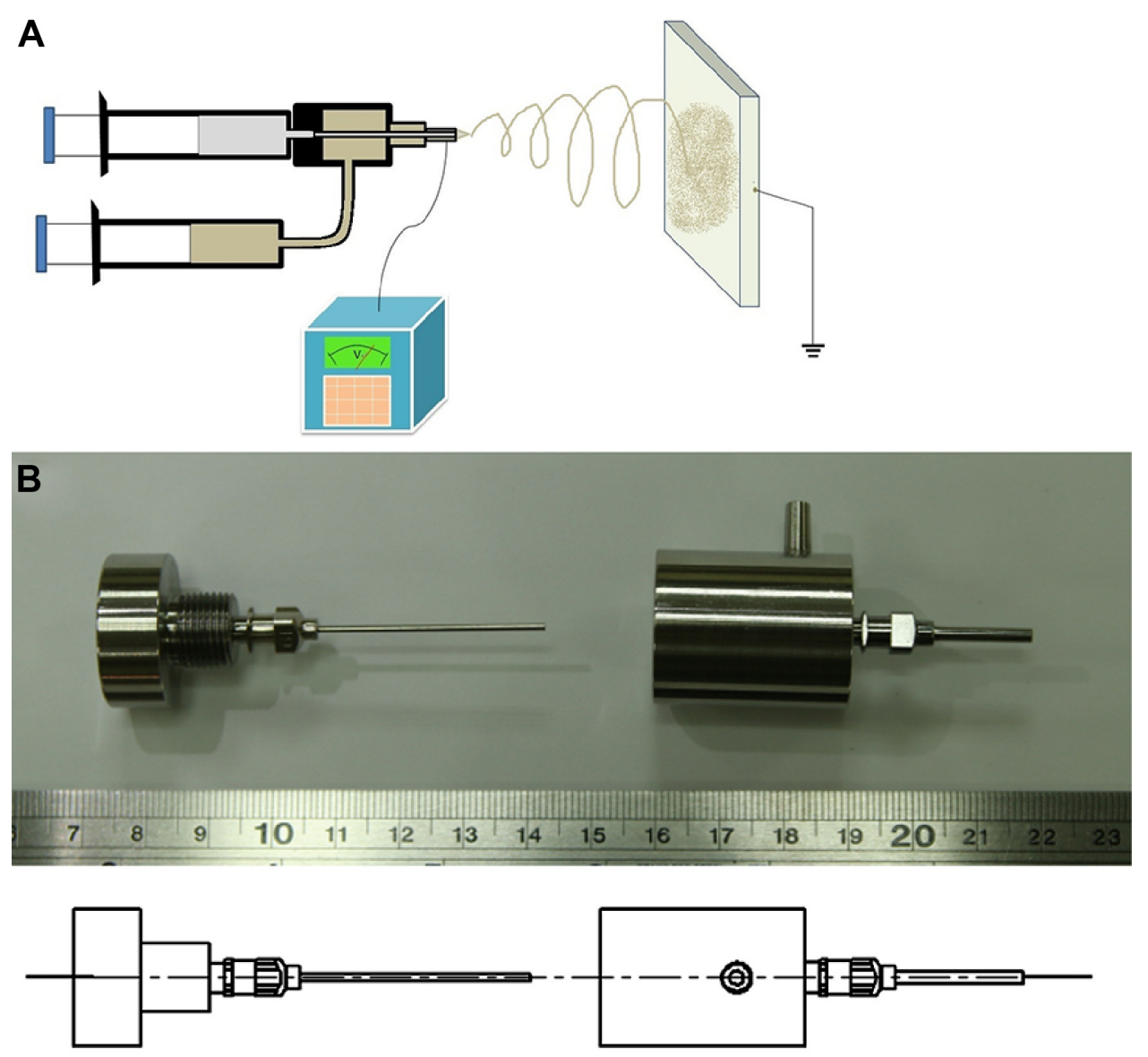

Figure I Fabrication process of sheath-core-structured nanofibers.

Notes: (A) Schematic of the experimental setup. (B) Photo of the device used to produce the sheath-core-structured nanofibers.

diameter and diameter distribution were obtained by analyzing SEM images using commercial ImageJ software (National Institutes of Health, Bethesda, MD, USA).

\section{Transmission electron microscopy}

The sheath-core-structured drug-eluting nanofibers were deposited on the copper network (PELCO ${ }^{\circledR}$ TEM, 01800-F, 200-mesh, Pelco Products Inc., Edmond, OK, USA) and observed by field emission transmission electron microscopy (JEM-2000EXII, JEOL).

\section{Laser scanning confocal microscopy}

To visualize the presence and distribution of proteins in the electrospun nanofibers, sheath-core-structured nanofibers with PLGA as the sheath and recombinant enhanced green fluorescent protein (reGFP) (6H1-38, Shanghai PrimeGene Bio-Tech, Shanghai, People's Republic of China) at the core were prepared. After co-electrospinning, we collected a thin layer of electrospun fibers on a glass slide. The fibers were observed using a laser-scanning confocal microscope (Leica TCS SP8X, Wetzlar, Germany). The excitation wavelength for reGFP was $487 \mathrm{~nm}$. Nanofibers of blended PLGA/reGFP were also observed for comparison.

\section{Contact angle measurement}

A water contact angle analyzer (First Ten Angstroms, Portsmouth, VA, USA) was employed. Specimens $(1 \times 1 \mathrm{~cm})$ were excised from electrospun nanofibrous membranes and placed on the testing plate, after which distilled water was dropped onto the membrane surfaces. The contact angles of virgin PLGA nanofibers, sheath-core drug-eluting nanofibers, and blended drug-eluting nanofibers were characterized using a camera.

\section{Tensile test}

The tensile properties of nanofibers were evaluated using a Lloyd tensile test machine (AMETEK, Berwyn, PA, USA). The uniaxial tensile tester was equipped with a 100-N load cell (ASTM D638). A membranous strip with dimensions $10 \times 50 \mathrm{~mm}$ was held between two clamps separated by $2 \mathrm{~cm}$. During measurement, the membrane was extended by the top clamp at a rate of $50 \mathrm{~mm} / \mathrm{min}$ through a distance of $10 \mathrm{~cm}$ until 
the strip fractured, at which point the maximum force and elongation were recorded. The measurements were performed in triplicate for each specimen. The ultimate tensile strength and the maximum elongation were calculated as follows:

$\underset{\text { Strength }(\mathrm{MPa})}{\text { Ultimate tensile }}=\frac{\text { Maximum force at fracture }(\mathrm{N})}{\text { Specimen cross - sectional area }\left(\mathrm{mm}^{2}\right)}$

$$
\begin{gathered}
\text { Maximum } \\
\text { elongation }(\%)
\end{gathered}=\frac{\begin{array}{c}
\text { Total increase in } \\
\text { length at fracture }(\mathrm{mm})
\end{array}}{\text { Original length }(\mathrm{mm})} \times 100 \%
$$

\section{Fourier transform infrared spectrometry}

A Fourier transform infrared (FTIR) spectrometry was employed to examine the spectra of virgin nanofibers and drug-loaded nanofibers. The FTIR analysis was conducted on a Bruker Tensor 27 spectrometer at a resolution of $4 \mathrm{~cm}^{-1}$ and 32 scans. Membrane samples were pressed as $\mathrm{KBr}$ disks, and spectra were recorded over the $400-4,000 \mathrm{~cm}^{-1}$ range.

\section{Standard curve}

HPLC analyses were conducted on a Hitachi L-2200 Multisolvent Delivery System. A SYMMETRY $\mathrm{C}_{8}$ column $(3.9 \mathrm{~cm} \times 150 \mathrm{~mm})$ was used to characterize vancomycin and ceftazidime (Waters, Milford, MA, USA). The mobile phase containing $0.01 \mathrm{~mol}$ heptanesulfonic acid (Fisher Scientific, Loughborough, UK) and acetonitrile (Mallinckrodt, St Louis, MO, USA) (85/15, v/v) was employed to separate vancomycin. The absorbance wavelength was set at $280 \mathrm{~nm}$, while the flow rate was $1.4 \mathrm{~mL} / \mathrm{min}$. The mobile phase used for ceftazidime contained methanol and $5 \mathrm{mM}$ phosphate buffer, pH 7.5 (Sigma-Aldrich) (10/90, v/v). The absorbance was monitored at $254 \mathrm{~nm}$ with a flow rate of $0.6 \mathrm{~mL} / \mathrm{min}$. All experiments were performed in triplicate $(\mathrm{N}=3)$.

\section{In vitro release of vancomycin and ceftazidime}

In vitro release of antibiotics from the nanofibrous membranes was evaluated by an elution method. Specimens $(10 \times 10 \mathrm{~mm})$ were excised from the electrospun membranes and placed in glass test tubes (one sample per test tube, $\mathrm{N}=3$ ) containing $1 \mathrm{~mL}$ phosphate-buffered solution (0.15 M, pH 7.4). The tubes were placed in an oven at $37^{\circ} \mathrm{C}$ for 24 hours before the solution was collected and replaced by fresh phosphatebuffered solution ( $1 \mathrm{~mL})$ for the next 24-hour period. The procedure was repeated for 35 days. The antibiotic concentrations in the collected solutions were characterized by HPLC using the constructed standard curves.

\section{Activities of released antibiotics}

The bioactivities of the released antibiotics were determined by a disk diffusion method (beef extract, peptone, Difco Laboratories, Detroit, MI, USA). Membrane eluent ( $8 \mu \mathrm{L}$ ) was pipetted onto the $6 \mathrm{~mm}$ absorption disks, and then the disks were placed on nutrient agar plates seeded with a layer of $S$. aureus (ATCC65389). The diameters of the inhibition zones were measured with a micrometer after incubating $16-18$ hours at $35^{\circ} \mathrm{C}$. A drug solution of $50 \mu \mathrm{g} / \mathrm{mL}$ was employed as the reference concentration. The bioactivity of the released vancomycin was defined as:

$$
\text { Bioactivity }(\%)=\frac{\begin{array}{c}
\text { Diameter of specimen } \\
\text { inhibition zone }
\end{array}}{\begin{array}{c}
\text { Diameter of reference } \\
\text { inhibition zone }
\end{array}} \times 100 \%
$$

The bioactivity of ceftazidime was determined using the same method against Escherichia coli (ATCC25922).

\section{BMP-2 detection by ELISA}

An ELISA (R\&D Systems, Inc., Minneapolis, MN, USA) was employed to characterize rhBMP-2 in the collected medium. A monoclonal antibody was first coated onto a 96-well microplate. The standards and the specimens were added to the wells, where rhBMP-2 was bound by the immobilized antibody. After washing away any unbound substances, an enzyme-linked monoclonal antibody specific for rhBMP-2 was added to the wells. Following a wash to remove any unbound antibody-enzyme reagent, a colorimetric substrate solution was added to the wells. The reaction was stopped with sulfuric acid, and the optical density change at $450 \mathrm{~nm}$ was recorded. Negative controls with media alone and positive controls with known concentrations of rhBMP-2 were included to facilitate construction of a standard curve. The concentration of rhBMP-2 in the samples was then determined by comparison to the standard curve.

\section{ALP assay}

Mesenchymal stem cells (rMSCs) were obtained from an 8 -week-old male SD rat. The Institutional Animal Care and Use Committee gave ethical approval for this study. The rat was sacrificed by $\mathrm{CO}_{2}$ asphyxiation following the guidelines of the Institutional Animal Care and Use Committee. The bone marrow (4-6 mL) was aspirated from the ventral ilium of SD rats (8 weeks old), and rMSCs were cultured as described by Lin et al. ${ }^{16}$ The stem cells were used for subsequent experiments. 
The P4 cells of rMSCs were cultured for 7 days with the eluents collected from the biodegradable sheath-corestructured drug-eluting nanofibers on days 1-30. Cultures were incubated in a humidified atmosphere of $5 \% \mathrm{CO}_{2} / 95 \%$ air until cell confluence. The medium was withdrawn, and a $10 \mathrm{~mL}$ aliquot of ALP substrate buffer $(50 \mathrm{mM}$ glycine, $1 \mathrm{mM}$ $\mathrm{MgCl}_{2}, \mathrm{pH} 10.5$ ) containing a soluble chromogenic ALP substrate ( $2.5 \mathrm{mM} p$-nitrophenyl phosphate) was added at room temperature. During incubation, cell-surface ALP converted $p$-nitrophenyl phosphate into $p$-nitrophenol, which then took on a yellow color. Twenty minutes after substrate addition, $1 \mathrm{~mL}$ buffer was removed from the culture and mixed with $1 \mathrm{~mL}$ of $1 \mathrm{~N} \mathrm{NaOH}$ to terminate the reaction. The absorbance of the mixture at $405 \mathrm{~nm}$ was measured in triplicate on an ELISA plate-reader (MRX, Dynatech Labs, El Paso, TX, USA) and compared with serially diluted standards. Enzyme activity was expressed as nanomole $p$-nitrophenol/min.

\section{Statistical analysis}

The data collected from the samples were analyzed using the paired sample $t$-test with commercially available SPSS software (version 12.0; SPSS Inc., Chicago, IL, USA). Data are expressed as mean \pm standard deviation, and the threshold for significance was set at $P<0.05$.

\section{Results \\ Characterization of electrospun nanofibers}

Both sheath-core-structured and blended drug-eluting nanofibers were successfully fabricated by the electrospinning technique. Figure 2 displays the SEM micrographs of the drug-eluting PLGA nanofibers under 10,000× magnification. The measured average diameters were $201 \pm 76$ and $436 \pm 159 \mathrm{~nm}$ for sheath-core-structured nanofibers and blended drug-eluting nanofibers, respectively, and the porosities of all fibrous membranes were high. Furthermore, the TEM image in Figure 3 demonstrates the sheath-core structure of electrospun nanofibers.

The tensile properties of electrospun nanofibers were evaluated. Figure 4 displays the measured results. The sheathcore-structured nanofibers exhibited somewhat lower tensile strength than the blended nanofibers (2.99 and 1.94 MPa,
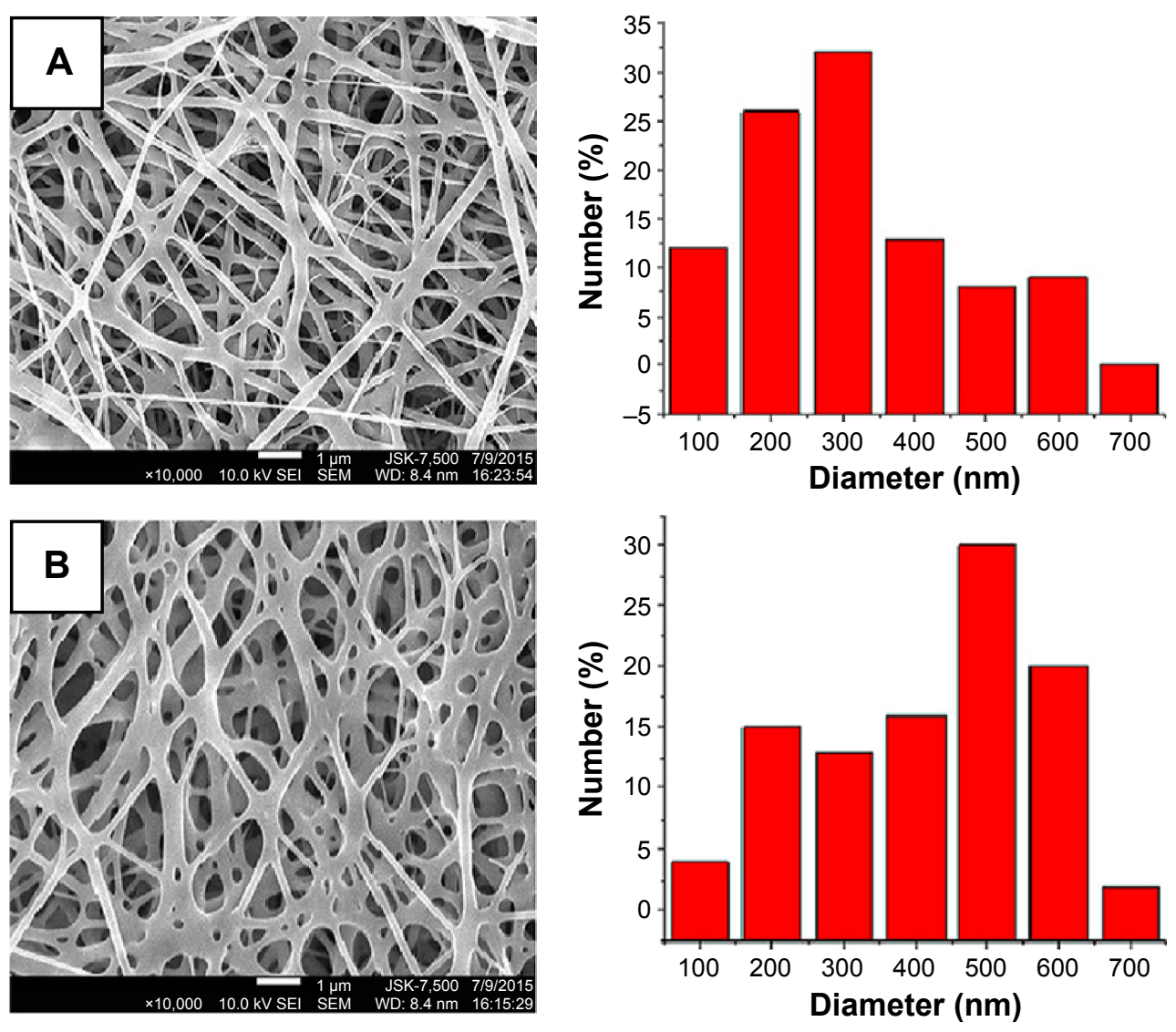

Figure 2 SEM micrographs of the drug-eluting PLGA nanofibers.

Notes: Morphologies and diameter distributions of (A) sheath-core-structured nanofibers and (B) blended nanofibers.

Abbreviations: SEM, scanning electron microscopy; PLGA, poly(D,L)-lactide-co-glycolic acid. 


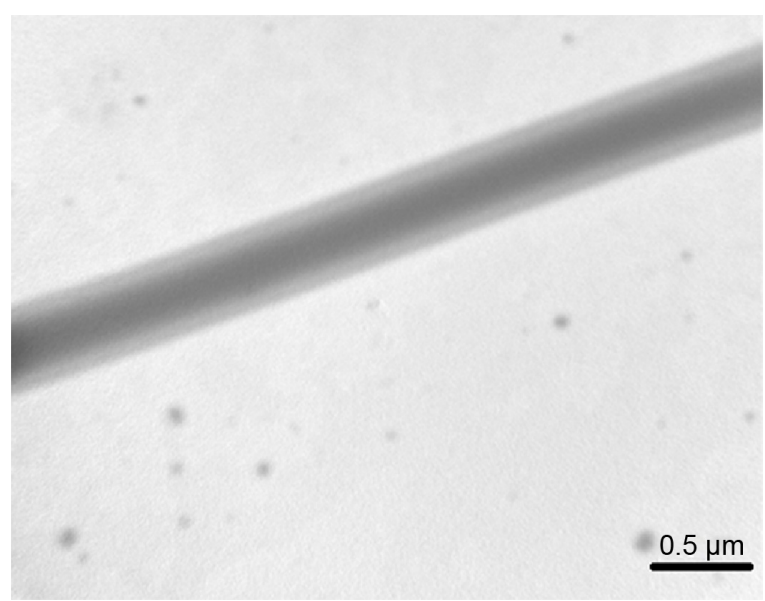

Figure 3 TEM image of sheath-core-structured nanofibers $(\times 60,000)$. Abbreviation: TEM, transmission electron microscope.

respectively), and the coaxially electrospun nanofibers showed greater elongation at breakage $(68.2 \%)$ than the blended nanofibers (37.2\%).

Figure 5 shows the water contact angles on electrospun nanofibrous membranes. The measured contact angles for virgin PLGA nanofibers, sheath-core-structured drug-eluting nanofibers, and blended drug-eluting nanofibers were $114.7^{\circ}$, $81.6^{\circ}$, and $82.3^{\circ}$, respectively. Clearly, the addition of antibiotics raised the hydrophilicity of the nanofibers.

The FTIR spectroscopy analysis was performed to verify the loaded drugs in the electrospun nanofibrous membranes. Figure 6 displays the measured spectra of virgin PLGA nanofibers and vancomycin/ceftazidime-loaded PLGA nanofibers. The absorption peaks of vancomycin/ceftazidime were observed in electrospun drug-eluting nanofibers. ${ }^{17,18}$ The results of FTIR spectra ${ }^{17,18}$ confirmed that the antibiotics were successfully embedded in the PLGA matrix.

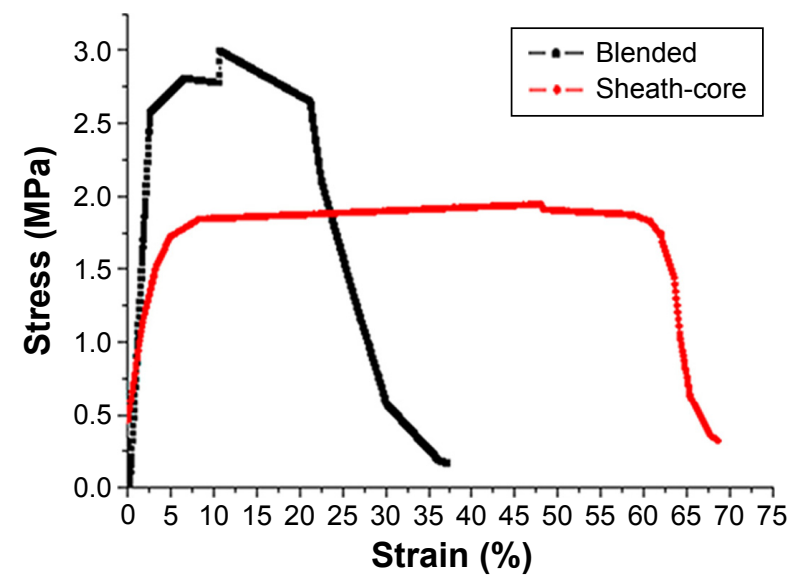

Figure 4 Tensile properties of electrospun nanofibers.
Laser-scanning confocal microscopy was used to visualize the protein distribution within the sheath-core and blended electrospun fibers. The coaxially electrospun sheath-corestructured fibers exhibited string-like green signals of reGFP (Figure 7A), indicating a relatively homogeneous protein distribution. In contrast, the blended nanofibers showed a bead-like appearance, with stronger green signals in the beads than in the fiber strings (Figure 7B). Since the sheath-corestructured nanofibers exhibited greater overall uniformity and higher protein activity after electrospinning, only the sheath-core drug-eluting nanofibers were investigated in subsequent experiments.

\section{In vitro release of drug-eluting nanofibers}

Figure 8 shows the measured daily and accumulated release curves of vancomycin and ceftazidime from the sheath-core drug-eluting nanofibers. Both vancomycin and ceftazidime exhibited similar release patterns characterized by an initially high release at day 1 , a second peak release after 5-10 days, and subsequently a more gradual and sustained release of the drugs. Moreover, the sheath-core drug-eluting nanofibers sustainably released high concentrations of vancomycin and ceftazidime (well above minimum inhibition concentration $[\mathrm{MIC}]_{90}$ ) for 32 and 35 days, respectively.

The bioactivities of eluted vancomycin on $S$. aureus (ATCC65389) and ceftazidime on E. coli were determined using an antibiotic disk diffusion method. Figure 9A and B show, respectively, the bioactivities of released vancomycin and ceftazidime, which ranged from $40 \%$ to $100 \%$. The antibiotic bioactivities remained high after the electrospinning process.

Figure 10 illustrates the ELISA-quantified released concentrations of rhBMP-2 over a period of 35 days for the sheathcore nanofibers loaded with 25 and $100 \mu \mathrm{g} / \mathrm{mL}$ rhBMP-2. Nanofibers loaded with 25 and $100 \mu \mathrm{g} / \mathrm{mL}$ rhBMP-2 showed sustained release of rhMBP-2 for 29 and 33 days, respectively. Furthermore, the nanofibers loaded with $100 \mu \mathrm{g} / \mathrm{mL}$ rhBMP-2 released more rhBMP-2 during elution.

The bioactivity of the released rhBMP-2 was determined by the ALP assay using nanofibers loaded with $25 \mu \mathrm{g} / \mathrm{mL}$ rhBMP-2. The experimental results demonstrate that the bioactivity of released rhBMP-2 remained high at all days (Figure 11), indicating that rhBMP-2 was not deactivated during the electrospinning process.

\section{Discussion}

The competing strategies of "union first" or "infection-elimination first" have been debated for a long time, and each has 


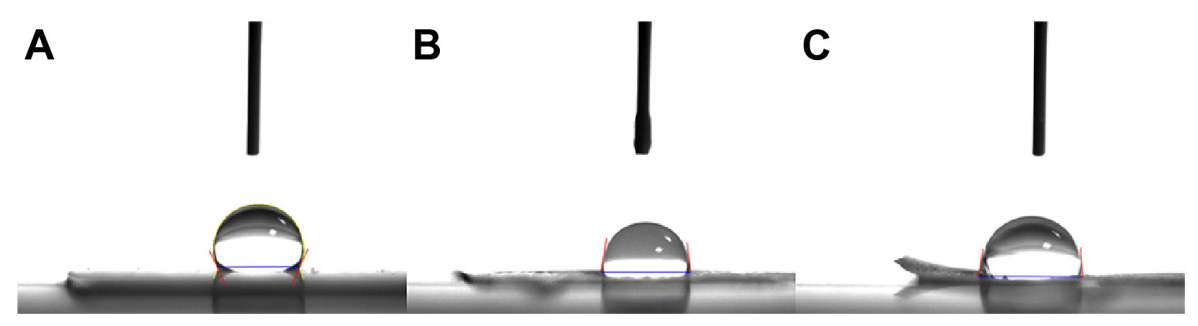

Figure 5 The water contact angles on electrospun nanofibrous membranes.

Notes: Water contact angles of $(\mathbf{A})$ virgin PLGA nanofibers $\left(114.7^{\circ}\right)$, (B) sheath-core-structured drug-eluting nanofibers $\left(81.6^{\circ}\right)$, and $(\mathbf{C})$ blended drug-eluting nanofibers $\left(82.3^{\circ}\right)$.

Abbreviation: PLGA, poly(D,L)-lactide-co-glycolide.

gained individual support in the study. ${ }^{19}$ To date, an integrated approach for good infection control and bone repair has not been established to our knowledge. In theory, any bone graft implanted into the bone defect of an infected nonunion must both prevent bacterial infection and promote bone ingrowth However, the release profile of antibiotics from drugs incorporated into auto or artificial bone grafts has been poor and may contribute significantly to development of multidrug-resistant bacteria. ${ }^{20}$ To combine the "union" and "infection-elimination" treatment strategies, we developed biodegradable sheathcore-structured nanofibers to offer sustained co-delivery of antibiotics (vancomycin and ceftazidime) and rhBMP-2 for infection treatment and bone regeneration.

Generally, the drug-release kinetics for biodegradable devices comprise three different phases: an initial burst, a diffusion-controlled release, and a degradation-controlled phase. During the fabrication process, most drugs are dispersed into the bulk of the PLGA matrix; however, certain pharmaceutical compounds may be located on the nanofiber surface, ultimately causing the initial burst. Following the initial burst, the drug-release profile is not only controlled

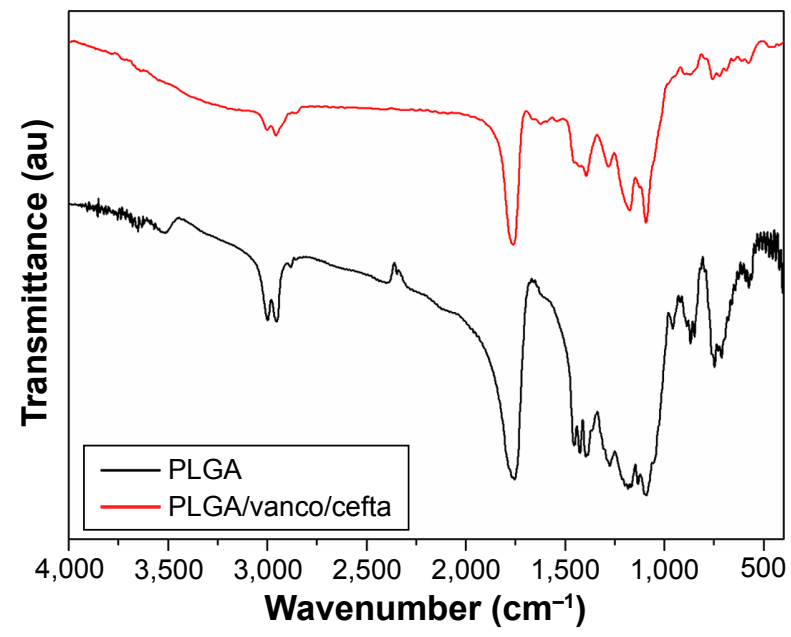

Figure 6 FTIR spectra of electrospun nanofibers.

Abbreviations: Cefta, ceftazidime; FTIR, Fourier transform infrared; PLGA, poly(D,L)lactide-co-glycolide; Vanco, vancomycin. by the diffusion effect, but also by polymer degradation. We therefore observed a relatively constant decrease in the release rate of vancomycin and ceftazidime. We further observed gentler and steadier release of rhBMP-2 at the core due to the barrier effect of the sheath PLGA. These results demonstrated that the biodegradable sheath-core-structured drug-eluting nanofibers released high concentrations of antibiotics/rhBMP-2 for over 4 weeks, which is advantageous for bone infection control and regeneration.

BMPs are known as a subgroup of growth factors that promote the bone healing process and tissue regeneration. ${ }^{6,7}$ At each fracture healing stage, different BMPs present different expression patterns. In particular, BMP-2 is important for chondrogenesis and osteogenesis. ${ }^{21,22}$ In the absence of BMP-2 expression, mice exhibit normal skeletal growth but impaired bone healing processes, as shown by Tsuji et al, ${ }^{23}$ indicating that other BMPs can still affect bone development but not bone healing. In this work, we showed that rhBMP-2 retention in scaffolds can be prolonged by employing the coaxial electrospinning technique to form sheath-corestructured nanofibers with vancomycin and ceftazidime at the surface layer. In vitro, the sustained release of rhBMP-2 in the core of electrospun nanofibers promoted osteogenic differentiation (Figure 9). The ability to deliver growth factors to the target site offers many possibilities for nanofibrous scaffold architecture and bioactivity. While the nanofibers released broad-spectrum antibiotics for infection control, the
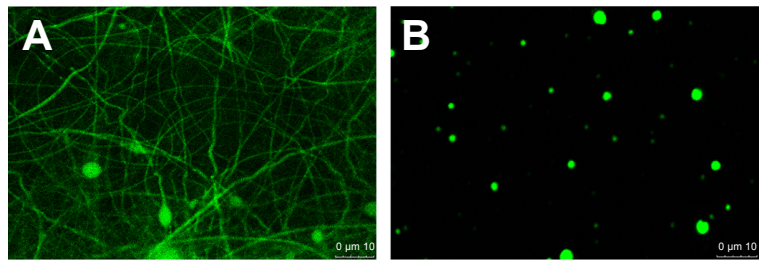

Figure 7 The protein distribution within the sheath-core and blended electrospun fibers.

Notes: Laser scanning confocal microscopy images of recombinant enhanced green fluorescent protein in (A) coaxial and (B) blended electrospun nanofibers. Magnification $\times 400$. 

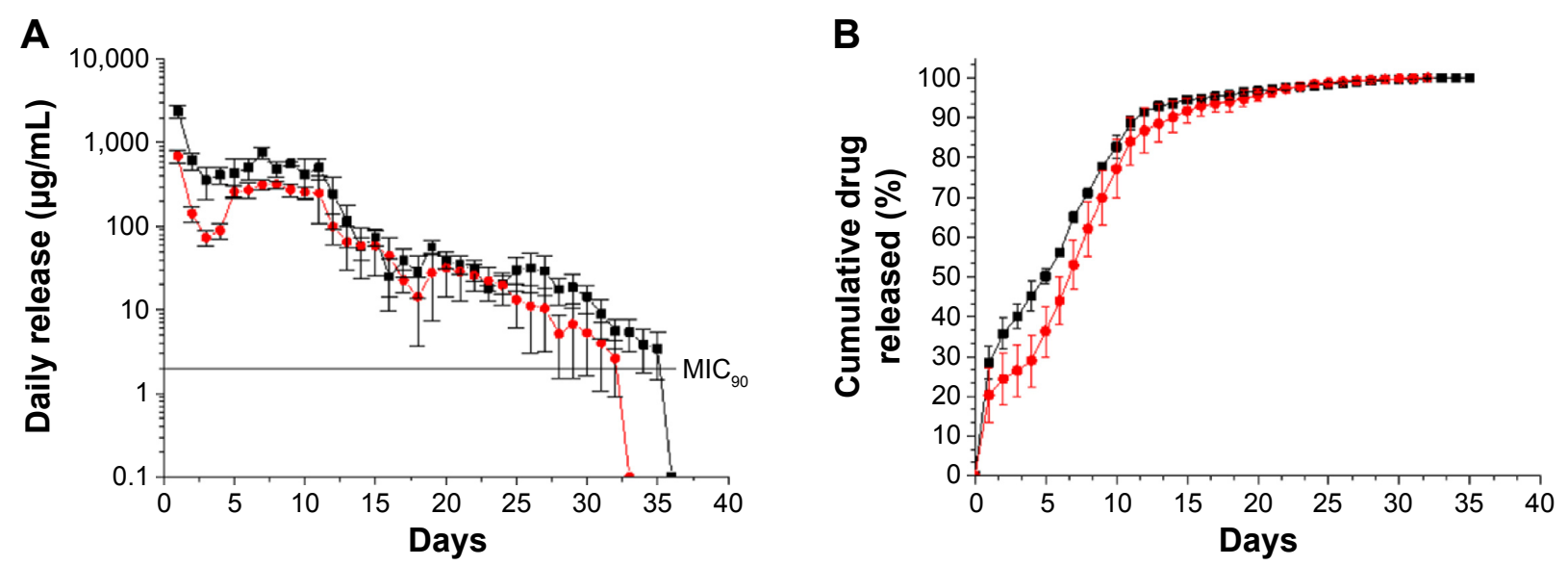

$-\mathbf{-}-$ Vancomycin $\quad--$ Ceftazidime

Figure 8 Daily and accumulated release curves of vancomycin and ceftazidime.

Notes: (A) Daily and (B) cumulative release of vancomycin and ceftazidime from the sheath-core-structured nanofibers.

steady presence of rhBMP-2 promoted bone regeneration in vitro for at least 4 weeks (Figure 8).

Many studies have investigated the release profile of antibiotics or growth factors. The innovation of BMP-2coupled nanosilver-PLGA composite grafts was introduced by Zheng et $\mathrm{al},{ }^{24}$ which improved the bone healing process in infected segmental bone defects. The defined nanosilver particle is bactericidal and does not interfere with the osteoinductive effect of BMP-2. The particles have been shown to have no in vivo and in vitro cytotoxicity. Given the earlier mentioned advantages, the nanosilver particles could be an ideal agent with bactericidal effects for promoting the bone healing process in infected wounds. Furthermore, to treat chronic osteomyelitis caused by methicillin-resistant S. aureus, Jia et a ${ }^{25}$ examined the efficacies of teicoplaninloaded calcium sulfate. Their results showed sustained release of teicoplanin and good bone regeneration with an antibiotic-impregnated calcium sulfate pellet. Nevertheless, they failed to identify the key role of bone fixation stability in achieving bone union while controlling the infection.

In our previous study, we demonstrated that the drugeluting nanofibrous membrane presented sustained release of vancomycin and ceftazidime with drug concentrations much higher than the $\mathrm{MIC}_{90}{ }^{8}$ Similarly, the biodegradable sheathcore-structured nanofibrous membranes in this study can also be attached to the surfaces of bone plates and screws for local drug release to the surrounding tissues without compromising stable fixation for segmental bone defect and further act as a carrier of artificial bone graft to the bone defect. This novel carrier not only prevented bacterial infection, but also promoted bone ingrowth.

Compared with the blended nanofibers, coaxially electrospun nanofibers have smaller cross-sectional areas because of their hollow cores, which correspondingly decrease the
A

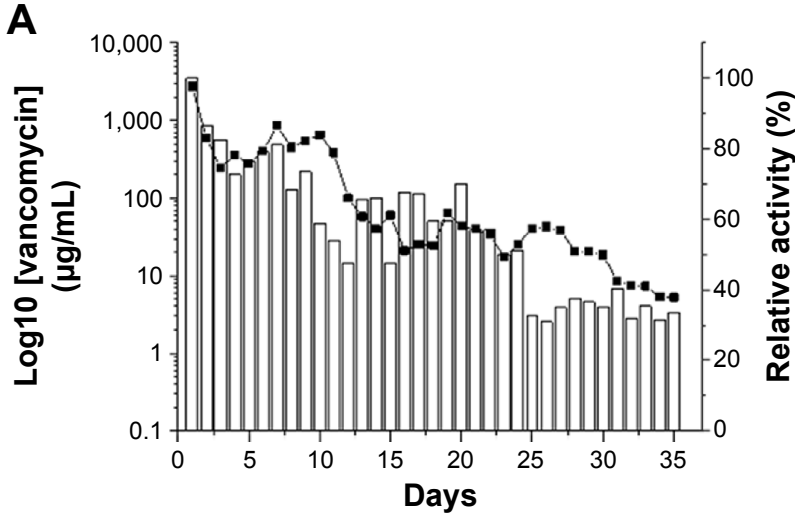

B

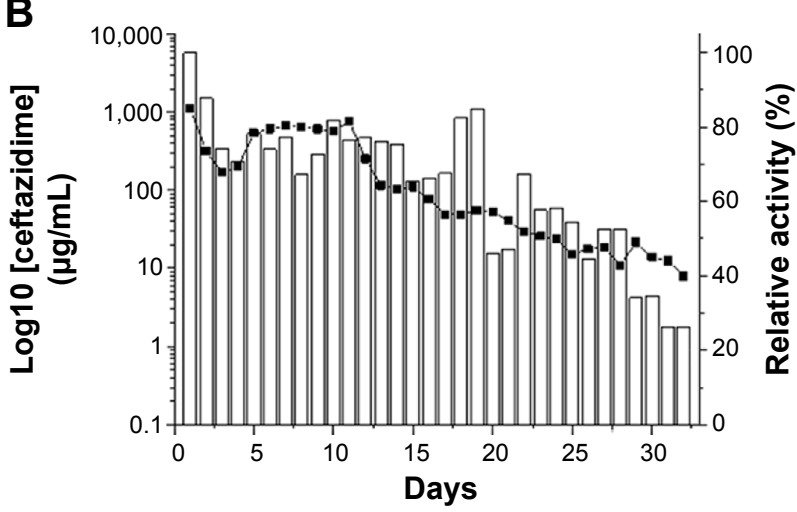

Figure 9 Bioactivity of eluted antibiotics.

Notes: (A) Activity of vancomycin against Staphylococcus aureus. (B) Activity of ceftazidime against Escherichia coli. 


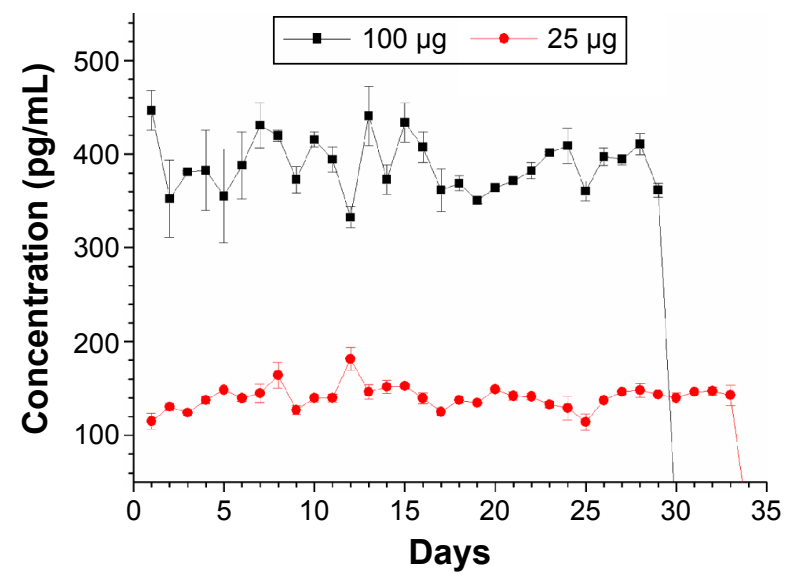

Figure 10 Release curves of rhBMP-2 on different days. Abbreviation: rhBMP, recombinant human bone morphogenetic protein.

mechanical strength. The smaller fiber diameter and the greater pore size distributions may also contribute to the less mechanical strength of the sheath-core nanofibers. Nevertheless, the sheath-core nanofibers exhibited greater elongations before breaking. This might be attributed to the proteins in the core providing a basis for flexibility and extensibility. Table 1 lists the comparison between the blended and sheath-core nanofibers. Additionally, a fiber with a smaller cross-sectional area can be stretched more during the electrospinning process. The fiber diameter thus decreased. Meanwhile, compared with virgin PLGA, antibiotics are hydrophilic materials. The presence of vancomycin and ceftazidime thus makes the electrospun nanofibrous membranes more hydrophilic.

The sheath-core nanofibers exhibited fiber sizes in the optimum range, that is, $350-1,100 \mathrm{~nm} \cdot{ }^{13}$ They might thus favor cell growth and promote healing. PLGA has been extensively applied in tissue engineering as a scaffold matrix

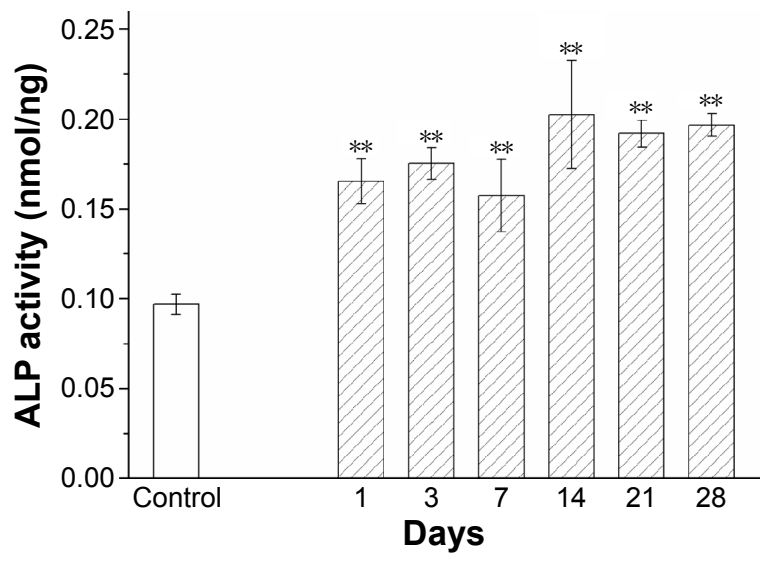

Figure I I Bioactivity of released BMP-2 on different days. Note: $* * P<0.01$.

Abbreviations: ALP, alkaline phosphatase; BMP, bone morphogenetic protein.
Table I A comparison of blended and sheath-core nanofibers

\begin{tabular}{lll}
\hline Property & $\begin{array}{l}\text { Blended } \\
\text { nanofibers }\end{array}$ & $\begin{array}{l}\text { Sheath-core } \\
\text { nanofibers }\end{array}$ \\
\hline Tensile strength & Higher & Lower \\
Elongation at break & Lower & Higher \\
Fiber diameter & Greater & Smaller \\
Fiber cross-sectional area & Greater & Smaller \\
Drug release pattern & Comparable & Comparable \\
Delivery of protein & No & Yes \\
\hline
\end{tabular}

material. Electrospun nanofibers have been employed as scaffolds in tissue engineering because of their distinct extracellular matrix that imitates a nonwoven microfibrous/nanofibrous architecture. ${ }^{26}$ By adjusting the manufacturing conditions, we were able to fabricate nanofibers with high porosity that were free of air bubbles or physical imperfections. ${ }^{27}$ HFIP, employed as the solvent, exhibits strong hydrogen bonding properties, allowing it to effectively dissolve various materials including polymers. ${ }^{28}$ The nanofibrous meshes were thus successfully electrospun using HFIP and exhibited desirable properties, including a high aspect ratio, a highly porous structure, and good mechanical strength, ${ }^{29}$ all of which are favorable for tissue healing and bone regeneration. Furthermore, the sheath-core-structured nanofibers showed excellent extensibility and flexibility. This supports the feasibility of their application for treating infection at bone joints while granting contraction of the tissue during healing.

Sustained-release and high local antibiotic concentrations contribute to infection control. Traditionally, during the treatment of osteomyelitis, a combination therapy of local drug release by PMMA carriers and systemic delivery is commonly adopted. Vancomycin and ceftazidime have been the antibacterial pharmaceuticals commonly employed with bone cement for osteomyelitis treatment. ${ }^{30,31}$ Herein, we fabricated a drug-eluting membrane with PLGA, rhBMP-2, vancomycin, and ceftazidime. Vancomycin is notorious for its renal toxicity; thus, a high concentration of vancomycin in the blood might raise safety concerns regarding high-dose antibiotic-eluting carriers. Springer et $\mathrm{al}^{32}$ reported that, out of 36 patients undergoing staged operation using vancomycingentamicin cement spacers for systemic safety evaluation, there were no systemic adverse effects of the antibiotics, and only one patient exhibited a transient rise in serum creatinine. Our previous in vivo animal study also demonstrated that the plasma creatinine level remains normal with delivery of high-concentration vancomycin at the fracture site. ${ }^{8}$ Herein, we demonstrated that biodegradable sheathcore-structured drug-eluting nanofibers could release high 
concentrations of vancomycin/ceftazidime (well above the $\mathrm{MIC}_{90}$ ) and rhBMP-2 for $>4$ weeks. After the electrospinning process, the experimental results also showed high level of bioactivities for the released vancomycin and ceftazidime..$^{8,10}$ Furthermore, the experimental results demonstrated that the fabrication process for the biodegradable sheath-corestructured nanofiber membranes was appropriate.

Finally, despite the promising in vitro results herein, we observe that further evaluation of the biodegradable sheathcore-structured nanofibers is necessary to elucidate their performance in vivo. Further, the efficacies of drug-eluting nanofibers in human infected segmental bone defect remain unclear and require further examination.

\section{Conclusion}

The PLGA sheath-core-structured drug-eluting nanofibers, which produce biocompatible hydrolysis products (lactic and glycolic acids) after biodegradation, released vancomycin and ceftazidime with similar release patterns characterized by an initially high release at day 1 , a second peak release after 5-10 days, and subsequently a more gradual and sustained release of the drugs. The bioactivities of released vancomycin and ceftazidime, which ranged from $40 \%$ to $100 \%$, remained high after the electrospinning process. Furthermore, the sheath-core drug-eluting nanofibers loaded with 25 and $100 \mu \mathrm{g} / \mathrm{mL}$ rhBMP-2 showed sustained release of rhMBP-2 for 29 and 33 days, respectively, and the bioactivity of released rhBMP-2 remained high for the whole study period. By adopting the electrospinning technique, it is possible to fabricate biodegradable nanofibers to carry various pharmaceuticals and proteins for sustained controlled drug release. The novel drug-eluting nanofibrous carrier can also integrate the strategies of "union" and "infection-elimination" for the treatment of osteomyelitis in long bone fractures.

\section{Acknowledgments}

The authors would like to thank the Ministry of Science and Technology, Taiwan (contract no 104-2221-E-182-048MY3), and the Chang Gung Memorial Hospital (contract no CMRPD3D0141) for financially supporting this study.

\section{Disclosure}

The authors report no conflicts of interest in this work.

\section{References}

1. Mader JT, Calhoun J, Cobos J. In vitro evaluation of antibiotic diffusion from antibiotic-impregnated biodegradable beads and polymethylmethacrylate beads. Antimicrob Agents Chemother. 1997;41:415-418.

2. Ueng SW, Wei FC, Shih CH. Management of femoral diaphyseal infected nonunion with antibiotic beads local therapy, external skeletal fixation, and staged bone grafting. J Trauma. 1999;46:97-103.
3. Hanssen AD. Local antibiotic delivery vehicles in the treatment of musculoskeletal infection. Clin Orthop Relat Res. 2005;437:91-96.

4. Hogan BL. Bone morphogenetic proteins in development. Curr Opin Genet Dev. 1996;6:432-438.

5. Nakase T, Yoshikawa H. Potential roles of bone morphogenetic proteins (BMPs) in skeletal repair and regeneration. J Bone Miner Metab. 2006; 24:425-433.

6. Struijs PA, Poolman RW, Bhandari M. Infected nonunion of the long bones. J Orthop Trauma. 2007;21:507-511.

7. Crowley DJ, Kanakaris NK, Giannoudis PV. Debridement and wound closure of open fractures: the impact of the time factor on infection rates. Injury. 2007;38:879-889.

8. Hsu YH, Chen DW, Tai CD, et al. Biodegradable drug-eluting nanofiber-enveloped implants for sustained release of high bactericidal concentrations of vancomycin and ceftazidime: in vitro and in vivo studies. Int J Nanomedicine. 2014;9:4347-4355.

9. Stewart S, Barr S, Engiles J, et al. Vancomycin-modified implant surface inhibits biofilm formation and supports bone-healing in an infected osteotomy model in sheep: a proof-of-concept study. J Bone Joint Surg Am. 2012; 94:1406-1415.

10. Chen DW, Hsu YH, Liao JY, et al. Sustainable release of vancomycin, gentamicin and lidocaine from novel electrospun sandwich-structured PLGA/collagen nanofibrous membranes. Int J Pharm. 430:335-341.

11. Stewart RL, Cox JT, Volgas D, et al. The use of a biodegradable, loadbearing scaffold as a carrier for antibiotics in an infected open fracture model. J Orthop Trauma. 2010;24:587-591.

12. Williams DF. Biodegradation of surgical polymers. J Mater Sci. 1982; 17:1233-1246.

13. Kumbar SG, Nukavarapu SP, James R, Nair LS, Laurencin CT. Electrospun poly(lactic acid-co-glycolic acid) scaffolds for skin tissue engineering. Biomaterials. 2008;29:4100-4107.

14. Ali SA, Doherty PJ, Williams DF. Mechanisms of polymer degradation in implantable devices. 2. Poly(DL-lactic acid).JBiomed Mater Res. 1993;27: $1409-1418$.

15. Kobayashi H, Shiraki K, Ikada Y. Toxicity test of biodegradable polymers by implantation in rabbit cornea. J Biomed Mater Res. 1992;26: $1463-1476$.

16. Lin CY, Chang YH, Lin KJ, et al. The healing of critical-sized femoral segmental bone defects in rabbits using baculovirus-engineered mesenchymal stem cells. Biomaterials. 2010;31:3222-3230.

17. Zarif MS, Afidah AR, Abdullah JM, Shariza AR. Physicochemical characterization of vancomycin and its complexes with $\beta$-cyclodextrin. Biomed Res-India. 2012;23:513-520.

18. Moreno ADH, Salgado HRN. Development and validation of the quantitative analysis of ceftazidime in powder for injection by infrared spectroscopy. Phy Chem. 2012;2:6-11.

19. Green SA, Dlabal TA. The open bone graft for septic nonunion. Clin Orthop Relat Res. 1983;180:117-124.

20. Kilgus DJ, Howe DJ, Strang A. Results of periprosthetic hip and knee infections caused by resistant bacteria. Clin Orthop Relat Res. 2002;404: $116-124$.

21. Onishi T, Ishidou Y, Nagamine T, et al. Distinct and overlapping patterns of localization of bone morphogenetic protein (BMP) family members and a BMP type II receptor during fracture healing in rats. Bone. 1998;22:605-612.

22. Kwong FN, Hoyland JA, Freemont AJ, Evans CH. Altered relative expression of BMPs and BMP inhibitors in cartilaginous areas of human fractures progressing towards nonunion. J Orthop Res. 2009;27:752-757.

23. Tsuji K, Bandyopadhyay A, Harfe BD, et al. BMP2 activity, although dispensable for bone formation, is required for the initiation of fracture healing. Nat Genet. 2006;38:1424-1429.

24. Zheng Z, Yin W, Zara JN, et al. The use of BMP-2 coupled - nanosilverPLGA composite grafts to induce bone repair in grossly infected segmental defects. Biomaterials. 2010;31:9293-9300.

25. Jia WT, Luo SH, Zhang CQ, Wang JQ. In vitro and in vivo efficacies of teicoplanin-loaded calcium sulfate for treatment of chronic methicillinresistant Staphylococcus aureus osteomyelitis. Antimicrob Agents Chemother. 2010;54:170-176. 
26. Toh YC, Ng S, Khong YM, et al. Cellular responses to a nanofibrous environment. Nano Today. 2006;1:34-43.

27. Meng ZX, Wang YS, Ma C, et al. Electrospinning of PLGA/gelatin randomly-oriented and aligned nanofibers as potential scaffold in tissue engineering. Mater Sci Eng C. 2010;30:1204-1210.

28. Zhang YZ, Su B, Venugopal J, Ramakrishna S, Lim CT. Biomimetic and bioactive nanofibrous scaffolds from electrospun composite nanofibers. Int J Nanomedicine. 2007;2:623-638.

29. Shin HJ, Lee CH, Cho IH, et al. Electrospun PLGA nanofiber scaffolds for articular cartilage reconstruction: mechanical stability, degradation and cellular responses under mechanical stimulation in vitro. $J$ Biomater Sci Polym Ed. 2006;17:103-119.
30. Chan YS, Ueng WN, Wang CJ, Lee SS, Chao EK, Shin CH. Management of small infected tibial defects with antibiotic-impregnated autogenic cancellous bone grafting. J Trauma. 1998;45:758-764.

31. Chang Y, Tai CL, Hsieh PH, Ueng SW. Gentamicin in bone cement: a potentially more effective prophylactic measure of infection in joint arthroplasty. Bone Joint Res. 2013;2:220-226.

32. Springer BD, Lee GC, Osmon D, Haidukewych GJ, Hanssen AD, Jacofsky DJ. Systemic safety of high-dose antibiotic-loaded cement spacers after resection of an infected total knee arthroplasty. Clin Orthop Relat Res. 2004;427:47-51.
International Journal of Nanomedicine

\section{Publish your work in this journal}

The International Journal of Nanomedicine is an international, peerreviewed journal focusing on the application of nanotechnology in diagnostics, therapeutics, and drug delivery systems throughout the biomedical field. This journal is indexed on PubMed Central, MedLine, CAS, SciSearch ${ }^{\circledR}$, Current Contents ${ }^{\circledR} /$ Clinical Medicine,

\section{Dovepress}

Journal Citation Reports/Science Edition, EMBase, Scopus and the Elsevier Bibliographic databases. The manuscript management system is completely online and includes a very quick and fair peer-review system, which is all easy to use. Visit http://www.dovepress.com/ testimonials.php to read real quotes from published authors.

Submit your manuscript here: http://www.dovepress.com/international-journal-of-nanomedicine-journal 